\title{
Rotating speed stability and mechanical vibrations analysis of a one-stage inertia flexible rotor driven by variable speed drives
}

\author{
F. Oliveira ${ }^{1,2}$, M. P. Donsión ${ }^{3}$, G. Peláez ${ }^{4}$
}

${ }^{1}$ Department of Electrical Engineering, School of Tecnology and Management, Polytechnic Institute of Leiria Campus 2 - Morro do Lena - Alto do Vieiro, 2411-901 Leiria, Apartado 4163, Portugal Phone: +351 244820 300, e-mail: ftadeu@estg.ipleiria.pt

${ }^{2}$ Institute for Systems and Computers Engineering at Coimbra Rua Antero de Quental, Nº199, 3000 - 033 Coimbra, Portugal Phone: +351239 851040

${ }^{3}$ Department of Electrical Engineering E.T.S.I.I., Vigo University

Campus of Lagoas - Marcosende, 36310 Vigo (Spain)

Phone/Fax number:+0034 986 812685, e-mail: donsion@uvigo.es

${ }^{4}$ Department of Mechanical Engineering, E.T.S.I.I., Vigo University gpelaez@uvigo.es

\begin{abstract}
This paper poses some questions as to the adequacy of the widespread use of variable speed drives. It presents a comparison of different variable speed drives, by studying their output wave forms and applying them to a mechanical one stage inertia flexible rotor specially designed for this purpose. The comparison of the output of the variable speed drives used reveals significant differences between them, which affect the way the load is driven and the overall behaviour of the mechanical system. A comparison against a sine wave reference shows that even the best of the variable speed drives used is outperformed, since the "perfect" sine wave ensures constant rotating speed, thus minimizing vibrations along the mechanical shaft.
\end{abstract}

\section{Key words}

Variable speed drives, power quality, mechanical vibrations

\section{Introduction}

Both the need for controllability and concerns on energy efficiency have led to the development and increasingly widespread use of variable speed drives (VSD) to control electric machines.

Where most of the times these devices adequately fulfil the stated objectives, power electronic components that constitute the base for these devices have a number of undesirable effects on power quality, namely harmonics and inter-harmonics.

Most of the variable speed drives presently available in the market provide a voltage wave controlled via a pulse- width modulation (PWM) or sinusoidal PWM, which only constitute square-wave approaches to the sine wave. Due to the inherent inductive behaviour of an induction motor, an adequately modulated sinusoidal PWM may provide a quasi-sinusoidal current wave, which for this purpose can be considered a sinusoidal wave "polluted" with harmonic content and high-frequency noise.

Squirrel-cage induction machines represent about $80 \%$ of electric motors used in industrialized countries. The model and behaviour of induction machines is thoroughly documented in both textbooks and numerous papers on the subject. Also, the relation between mechanical torque, rotating machine, input voltage and frequency are also well known.

An increase in mechanical vibrations, components wear, heating, and general lifetime decrease are some of the consequences of poor power quality input to a motor. However, most models available assume perfect sinusoidal voltage wave input, perfectly balanced phases and a simple but incomplete model for the mechanical loads.

Hence, the work presented in this paper concentrated on the actual behaviour of induction machines and mechanical loads driven by different variable speed drives.

\section{Laboratory equipment and tests}

In order to study the influence of the electric drive on the 
overall behaviour of a mechanical load, a one-stage inertia flexible rotor (OSIFRO) set was specially designed, dimensioned and built. This provided a simple mechanical load with a well-fitted (and well-known) dynamic model with which to work. Figs. 1 and 2 show a view of the OSIFRO model and a representation of the rotor shaft on the bearings:

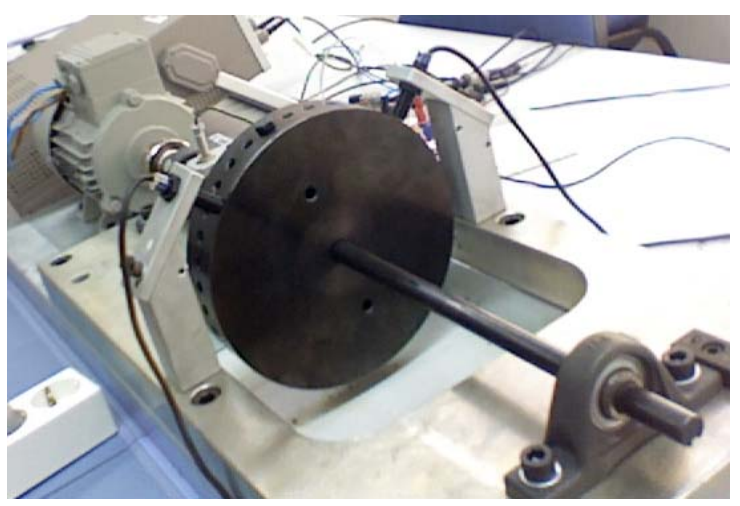

Fig. 1. A view of the OSIFRO model

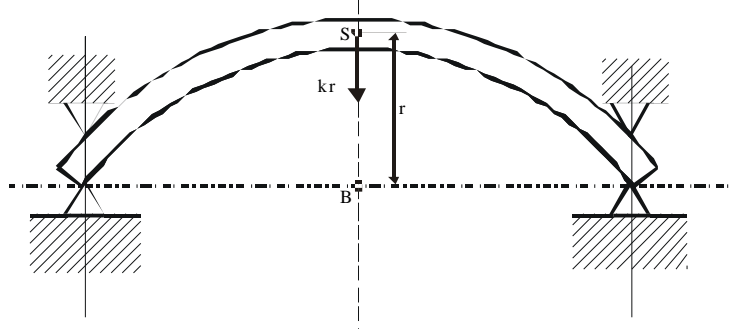

Fig. 2. A representation of the flexible rotor shaft on the bearings

To drive the OSIFRO, a 0,37 $\mathrm{kW}$ squirrel-cage induction machine was connected using three different equipments:

1) A single-phase input, three-phase (PWM) output, Siemens 0,37 kW VSD - VS1.

2) A three-phase input, three-phase (sinusoidal PWM) output, TeleMecanique Altivar 71, 1,5 kW VSD - VS2

3) A three-phase input, $15 \mathrm{kVA}$ programmable AC power source from California Instruments, which in this instance provided perfect voltage sine waves with a range of amplitudes and frequencies, and was used as a reference to the tests performed with the VSDs

The OSIFRO mechanical load is constituted by a flexible rotor with a cylindrical inertia. This mechanical load is intrinsically slightly unbalanced, and adequate correction masses can be added to balance the rotor.

This work required a number of electrical and mechanical quantities to be measured. In order to acquire and accommodate the several quantities to be measured, a PC-based, high-precision data acquisition card was used, together with voltage and current active probes, accelerometers, proximity inductive sensors and a keyphasor.

\section{Variable speed drives - voltage and current waveform}

Variable speed drives are generically constituted by three stages:

1) Rectifier bridge

2) DC bus

3) Three-phase inverter bridge

As far as the motor is concerned, the decisive stage is the inverter bridge. The wave forms in each stage for a standard PWM output VSD are depicted in Fig. 3.

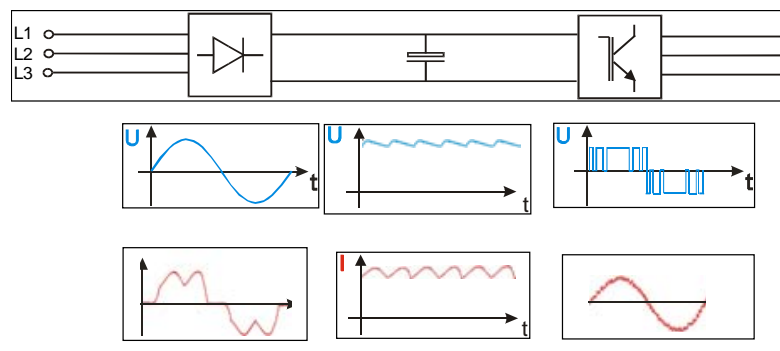

Fig. 3. Typical voltage and current waveforms on the various stages of a variable speed drive

The first part of the this work was intended to determine the differences between common, mainstream small variable speed drives, to allow later comparison of mechanical effects.

The PWM-output VSD1 presents an output voltage wave constituted by a set of higher-frequency variable-width semi-square waves, which approximate a lowerfrequency modulated square-wave. When applied to the motor, the resulting current is an approximation of a modulated sine-wave, with considerable higherfrequency noise. Figures $4 \mathrm{~A}$ to $4 \mathrm{C}$ show actual voltage and current measurements obtained with a high precision and adequate sampling rate instrument.

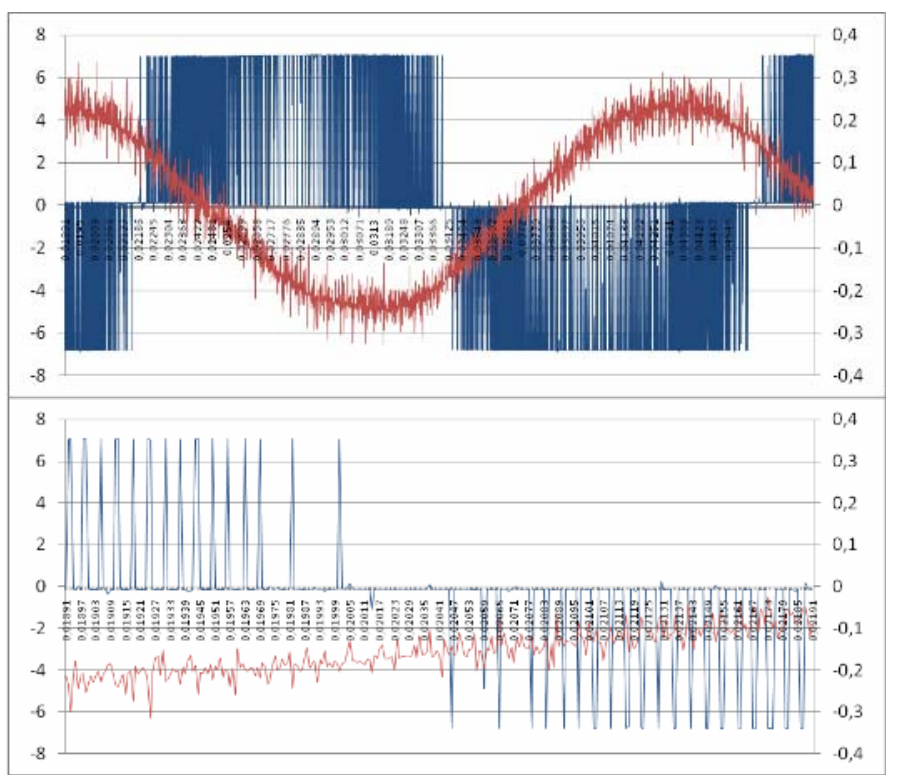




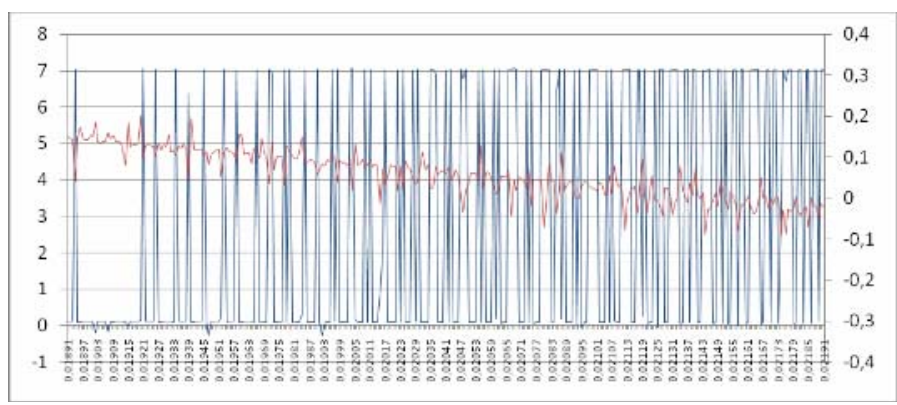

Figs. 4A to 4C.

A) VS1 output @ 20 Hz, approx. one period;

B) and C) 10x zoom-in.

The tests performed with sinusoidal-PWM output VSD2 revealed a wave form similarly built, although the approximation to the sine wave was significantly better. This applies mainly to the current wave, which, while being similar to the former case, presented less "noise".

The programmable power source provided near-perfect voltage sine waves to the motor, and was therefore used as a reference for this test. In order to have a comparable approach, the sine wave output was adjusted so that the rotating speed of the machine was the same as that provided by VSD1 and VSD2.

\section{Mechanical vibrations and rotation speed}

A one-stage inertia flexible rotor can be modelled as a one degree of freedom system, and thus has one vibration mode. This is obviously a simplified model, yet an effective one for this purpose.

This results in an orbit-like motion of the shaft around its central axis, which will be measured using the inductive proximity sensors installed in the OSIFRO, as depicts Fig. 5:

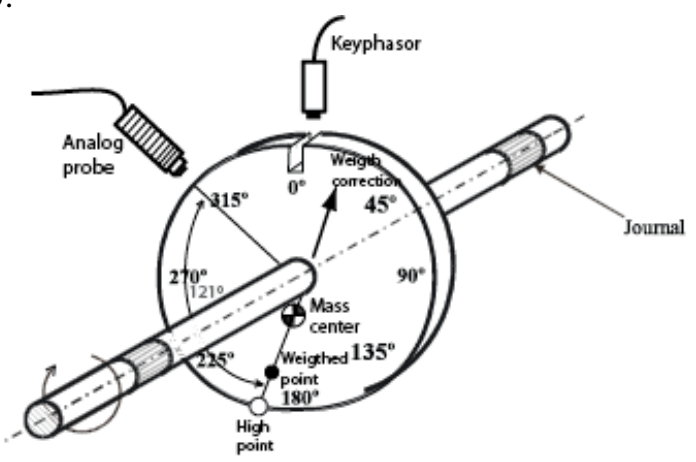

Fig. 5. A schematic of the OSIFRO, showing one of the analog probes measuring the distance to the shaft.

Although the mechanical modelling of the rotor is beyond the scope of the present paper, it can be stated that the resolution of the motion equations provides as oscillating system, with a natural frequency of around 23 $\mathrm{Hz}$.

Also, because the OSIFRO shaft has a slot along its length, the elasticity is variable, causing a subcritical natural frequency to occur.
Figures $6 \mathrm{~A}$ and 6B show the response of OSIFRO driven by VSD1:
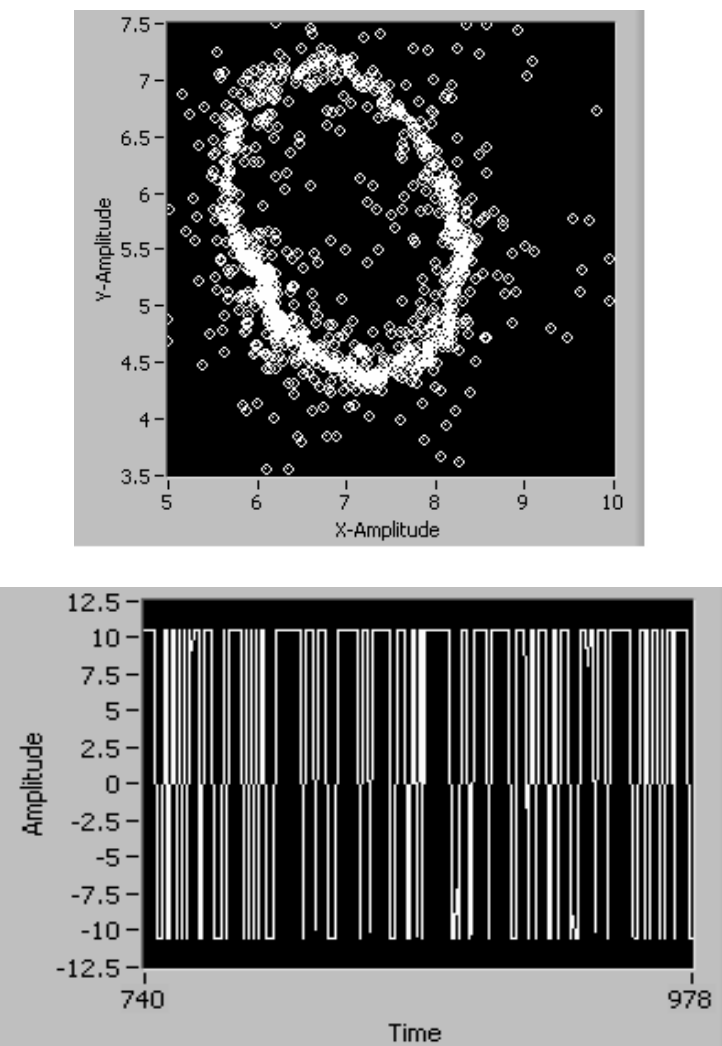

Figs. 6A and 6B.

A) Shaft orbit, VS1@15 Hz;

B) Keyphasor signal, VS1@15 Hz.

It is clear from Fig. 6A that the rotor shaft vibrates, producing a wide, approximately elliptic orbit. Fig. 6B clearly shows that, using VSD1, rotating speed is very unsteady in time.

Figs. 7A and 7B depict the response of the same system, this time driven by VS2:

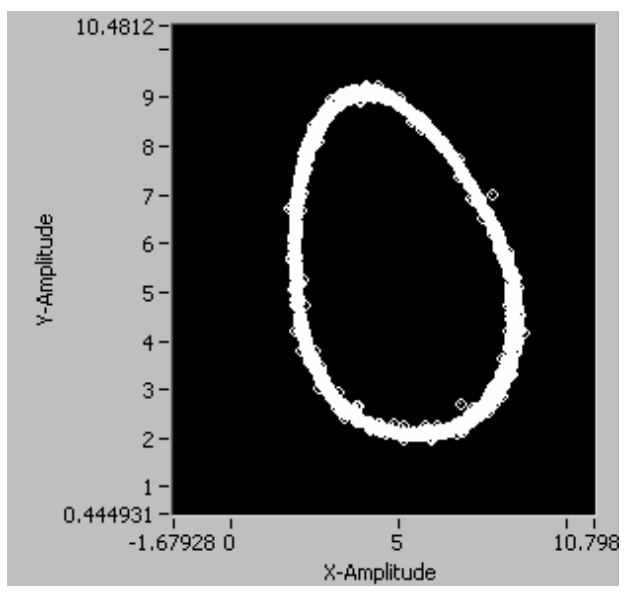




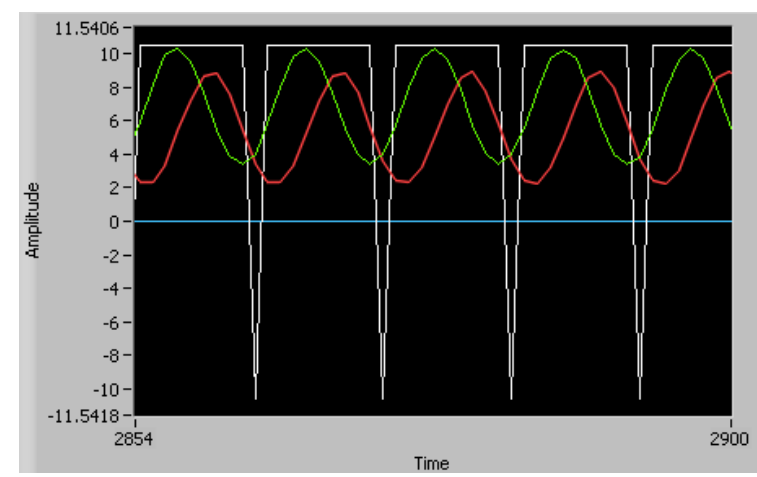

Figs. 7A and 7B.

A) Shaft orbit, VS2@20 Hz;

B) Keyphasor and prox. sensors signals, VS2@20 Hz.

Fig. 7A shows a steadier orbit when compared with Fig. $6 \mathrm{~A}$, due to the better quality of the voltage wave. Fig. 7B shows a detail of the keyphasor signal, revealing a steady rotating speed, and the proximity sensors (placed orthogonally over the shaft).

In order to have a term of comparison, Figs. 8A and 8B depict the system response when the input voltage is fed using the programmable voltage source:
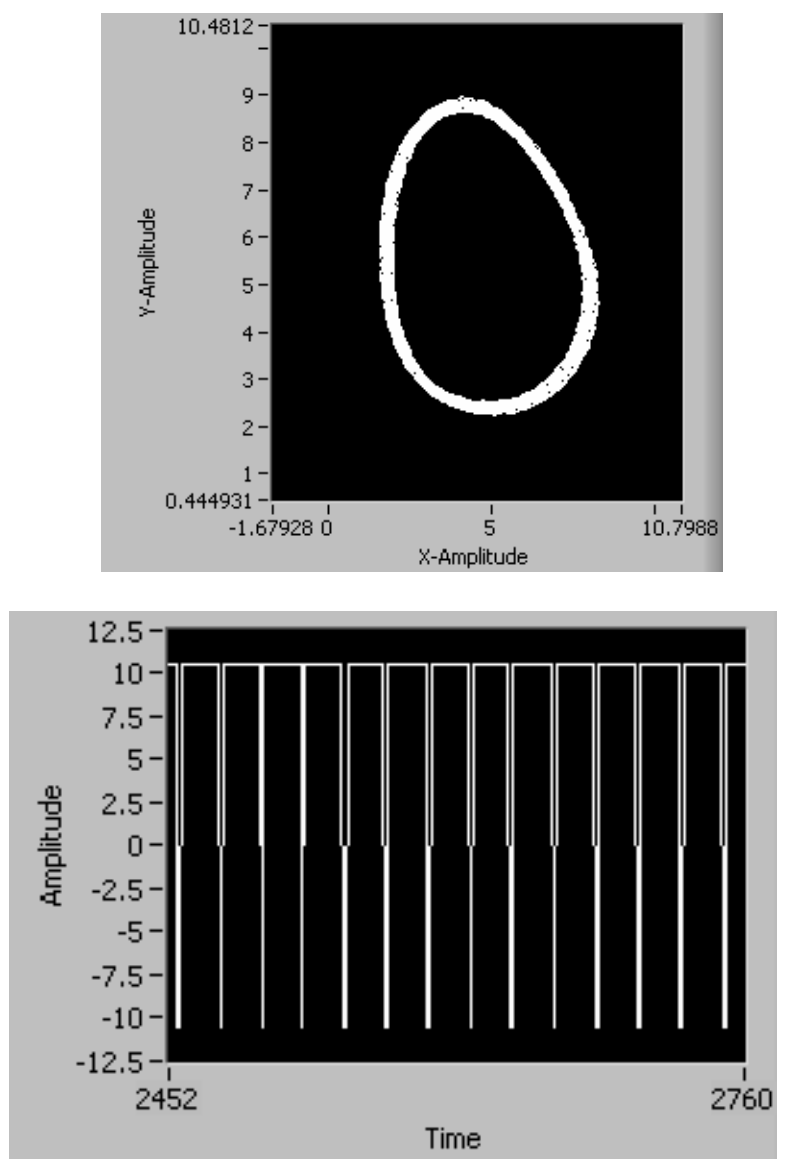

Figs. 8A and 8B.

A) Shaft orbit, VS2@20 Hz;

B) Keyphasor signal, VS2@16 Hz.
Fig. 8A shows a near-perfect orbit, suggesting constant rotating speed; fig. $8 \mathrm{~B}$ confirms it, as the keyphasor signal is constant in time.

\section{Conclusions and Outlook}

Throughout this work, a number of commonly accepted and widespread ideas about the virtues and uses of variable speed drives have been shaken or even proven wrong.

While it is clear that variable speed drives allow an easy and more flexible use of an induction motor, results show that uses where rotating speed stability is important may be incompatible with these devices.

The results also show that there are important differences between the various variable speed drives, and that the information provided by the manufacturers is manifestly insufficient to predict if rotating speed stability is sufficient.

The study of a simple mechanical system also allows the authors to conclude that the use of variable speed drives may cause or increase undesirable effects, such as higher amplitude vibrations, heating and overall lifetime reduction of machinery.

\section{Acknowledgement}

The authors wish to acknowledge the financial support of Spanish government through "Ministerio de Ciencia e Innovación” research project ENE2007-6803-C04-01.

\section{References}

[1] Bollen, M. H. J. on Power Engineering, I. P. S., ed., "Understanding Power Quality Problems, Voltage sags and interruptions", Wiley - Interscience, A John Wiley \& Sons, Inc., 2000.

[2] J.P. Den Hartog, "Mechanical Vibrations", MacGraw-Hill 4th ed. New York, 1956 (Reprint).

[3] Khan, S.; El-Dehaibi, N. "System and equipment problems associated with variable frequency drives in the pulp and paper industry", Pulp and Paper Industry Technical Conference, 1995., Conference Record of 1995 Annual, 12-16 Jun 1995 Page(s):131 - 136 